\title{
Rapid Isolation of intact Salmonella-containing vacuoles using paramagnetic nanoparticles
}

Vikash Singh ${ }^{1,2^{*}}$, Peter Schwerk ${ }^{1}$ and Karsten Tedin ${ }^{1 *}$

\begin{abstract}
Background: Both typhoidal and non-typhoidal Salmonella infections remain a considerable cause of morbidity and mortality globally, and impose a major socio-economic burden worldwide. A key property of all pathogenic Salmonella strains is the ability to invade host cells and reside within an intracellular, vacuolar compartment called the Salmonella-containing vacuole (SCV). Although the SCV is involved in both immune-evasion and intracellular replication and spread within the host, information about the host:pathogen interactions at this interface are limited, in part due to the technical difficulties involved in purification of these vacuoles. While a number of column- or gradientbased methods have been applied, cross-contamination with other host cell organelles or rupture of the labile SCV membrane has further complicated efforts to successfully isolate SCVs.
\end{abstract}

Results: Here, we report the isolation of intact SCVs using carbon-coated, paramagnetic nanoparticles. The approach permits rapid isolation of intact SCVs from human macrophages in vitro without involving numerous purification steps. Bacteria are pre-labeled with modified nanoparticles prior to infection, and at various times post-infection, host cells are lysed and intact pathogen-containing phagosomes are recovered after application of a mild magnetic field. Purified, intact SCVs isolated using this method were shown to display high levels of co-association of internalized Salmonella with the standard SCV markers Rab5 and LAMP-1 using both microscopic and protein based methods.

Conclusion: The method described is highly efficient, robust and permits rapid isolation of intact SCVs from human macrophages without involving numerous purification steps. The method can also be applied to other intracellular pathogens that reside within a vacuole-like compartment within host cells. Future work using the approach should aid in identification and characterization of host factors associated with the membranes of such intracellular pathogens, which could potentially serve as pharmaceutical targets against intracellular pathogens residing within vacuoles.

Keywords: Nanoparticles, Bacteria containing phagosome, Isolation, Salmonella, Infection, Macrophages

\section{Background}

Salmonella enterica serovars are Gram-negative, facultative intracellular bacteria that infect and colonize vertebrate hosts with outcomes ranging from sub-clinical infections to life-threatening systemic diseases

\footnotetext{
*Correspondence: vikash.singh@fu-berlin.de; vs399@cam.ac.uk; karsten. tedin@fu-berlin.de

${ }^{1}$ Centre for Infection Medicine, Institute of Microbiology and Epizootics, Free University of Berlin, Robert-von-Ostertag-Str. 7-13, 14163 Berlin, Germany

${ }^{2}$ Department of Pathology, University of Cambridge, Tennis Court Road, Cambridge CB2 1QP, UK
}

[1]. Salmonella infections constitute a global public health burden, not only due to infections by $S$. Typhi, the human-restricted serovar causing human Typhoid, but also due to non-Typhoidal salmonellosis. The nontyphoidal Salmonella serovars can differ both in their host specificity and in the pathogenesis in different species [2]. The broad host-range serovar Salmonella enterica serovar Typhimurium infects a wide range of hosts, both human and animal, as well as different host cell types, including macrophages and non-phagocytic cells such as the intestinal epithelia $[3,4]$.

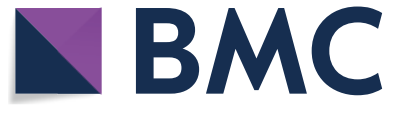

(c) The Author(s) 2018. This article is distributed under the terms of the Creative Commons Attribution 4.0 International License (http://creativecommons.org/licenses/by/4.0/), which permits unrestricted use, distribution, and reproduction in any medium, provided you give appropriate credit to the original author(s) and the source, provide a link to the Creative Commons license, and indicate if changes were made. The Creative Commons Public Domain Dedication waiver (http://creativecommons.org/ publicdomain/zero/1.0/) applies to the data made available in this article, unless otherwise stated. 
The major Salmonella virulence genes involved in host cell invasion and establishment of the intracellular replicative compartment known as the Salmonella-containing vacuole $(\mathrm{SCV})$ are encoded within two pathogenicity islands, denoted SPI-1 and SPI-2, respectively $[5,6]$. Salmonella translocates more than 30 SPI-2 encoded effectors or virulence factors across the $\mathrm{SCV}$ membrane, which enable the pathogen to replicate, colonize and establish infection within its host [7]. The maturation of the SCV is characterised by a time-dependent acquisition and loss of various endosomal and lysosomal markers that distinguish it from the lysosomal degradation pathway $[8,9]$.

A major difficulty in identifying and understanding the various host proteins involved in maturation of pathogen-specific, phagosome-like compartments has been the isolation of intact pathogen-containing phagosomes from host cells. Bouyant density gradient centrifugation has been previously applied, in which latex beads are pre- or post-loaded with phagosomes containing pathogens and purified from fractions taken from the gradients where the latex particles show a discrete banding pattern [10, 11]. However, this method is dependent on separation from within a gradient of vesicles with similar densities, where cross-contamination is often unavoidable. Magnetic cell separation (MACS) and other column-based methods require removal of non-bound material with extensive washing steps, and labile compartments such as phagosomes may lose considerable amounts of material and loosely bound proteins $[12,13]$. Uncertainties due to cross-contamination with other cellular compartments or numerous purification steps accompanied by loss of the labile vacuolar membrane has therefore hampered a better characterisation of these intracellular compartments. An improvement has been the application of flow-assisted cell sorting (FACS), in which GFP- or other fluorescently-labelled bacteria are used to infect cells, and after lysis of the cells, the cellular contents are passed through a flow cytometer and fluorescently-labelled components are diverted from the flow-through for collection [14]. However, the shear forces generated often lead to disruption of the phagosomes and the volumes required are often accompanied by inefficient concentration of the samples.

Here, we describe a simple method for successfully isolating intact SCVs by pre-labelling the bacteria with carboxyl-coated paramagnetic nanoparticles prior to infection. The method described is rapid, robust and efficient enough to collect intact SCVs for further, downstream analyses such as Western blotting, immunofluorescence, and intravacuolar bacteria recovery.

\section{Results}

\section{Overview of the isolation method}

The methodology described for the isolation of intact Salmonella-containing vacuoles (SCVs) makes use of paramagnetic nanoparticles that are between 10 and $50 \mathrm{~nm}$ in diameter. The nanoparticles consist of a central metal cobalt ion, which is carbon-coated to avoid possible metal toxicity, and further modified with carboxyl functional groups to provide a charge for non-covalent binding to the bacterial (target) surface.

Unbound nanoparticles are removed by passing the mixture through a $0.2 \mu \mathrm{m}$ filter, which allows free passage of non-bound nanoparticles. After washing of the retained bacteria, the filter is inverted and the nanoparticle-tagged bacteria are recovered by a back wash with buffer. The recovered bacteria are then used to infect host cells using standard gentamicin-protection (invasion) assays [15]. At different time points post-infection, the infected host cells are lysed and bacteria are recovered in the presence of a magnetic field. An overview of the method is shown in Fig. 1. The recovered bacterial samples are then stained with antibodies specific to host cell proteins known to co-localise with the SCV membrane such as Rab5, EEA1 or LAMP-1 to verify the presence of intact vacuolar membranes prior to further characterisation.

\section{Non-covalent attachment of carboxyl-coated magnetic nanoparticles to the bacterial surface}

Bacterial attachment to any surface is related to surface charges both on the bacteria as well as on the substratum or target of attachment. Dickson et al. [16] characterized surface charges and their correlation with bacterial attachment. In that study, it was found that for Salmonella Typhimurium, the bacterial surface showed an overall net negative charge with $\mathrm{r} / \mathrm{e}(-) 9.47$ to $\mathrm{r} / \mathrm{e}$ (+) 4.78, consistent with earlier studies [17]. Therefore, although the bacterial cell surface has generally an overall negative net charge, there are regions of positive charge present as well. We considered that an excessive labeling of the exterior surface of the bacteria with positively charged particles might result in detrimental effects on either host cell invasion or cytotoxic effects on the host cells. Although the nanoparticles used are much smaller than the average bacterial cell $(1-2 \mu \mathrm{m})$, excessive particle binding to the bacteria could affect the function of the type III secretion systems, or Salmonella's natural trafficking within the cell. We therefore chose to take advantage of the small, but significant, positive charges on to the bacterial surface to label the bacteria. For this purpose, we used paramagnetic, carbon-coated cobalt nanoparticles of diameter $10-50 \mathrm{~nm}$, with a carboxyl functional group attached to their surface, thus providing 

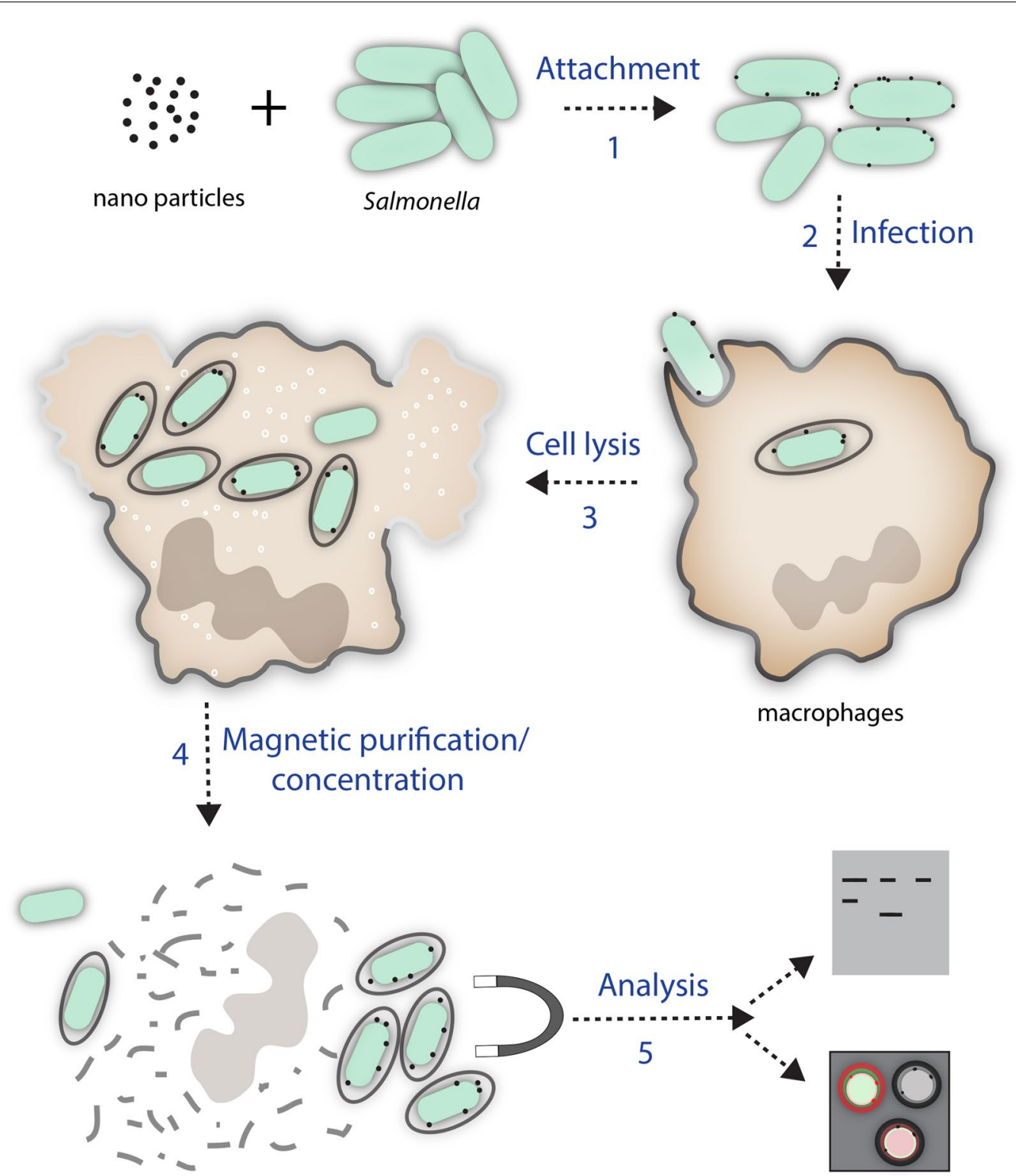

Fig. 1 Overview of the method. Carboxyl-coated paramagnetic nanoparticles are incubated with bacteria for 30 min with shaking (1). The paramagnetic nanoparticle-labelled Salmonella were then used to infect the human macrophage like cell line THP-1. The internalization of the bacteria is synchronized by a short centrifugation step (2). Internalized Salmonella establish an intracellular SCV (3). At different time points, macrophage are lysed using a mild detergent containing $2 \%$ sucrose solution (4), and the bacteria are recovered by application of a magnetic field (5). The recovered bacteria are then analysed by standard methods such as immunofluorescense and Western blotting for the presence or absence of standard SCV markers

a net negative charge for attachment via electrostatic interactions [18] to positively charged regions on the cell surface of Salmonella. A schematic representation for the attachment is shown in Fig. 2.

Carboxyl-coated nanoparticles were first sonicated to disperse aggregates and incubated with bacteria suspended in 1× PBS, at a ratio of approximately 5:1 of nanoparticles:bacteria at $37{ }^{\circ} \mathrm{C}$ for $20 \mathrm{~min}$ with shaking.
The bacterial suspensions were then filtered through a $0.2 \mu \mathrm{m}$ filter fitted to a $5 \mathrm{ml}$ syringe. The filters were washed twice with two volumes of PBS to remove unbound nanoparticles. The filters retaining the bacteria were then inverted and the bacteria were recovered in $1 \mathrm{ml}$ of PBS collected into a sterile microfuge tube. The recovered bacteria were then used for either infection of cell cultures of THP-1 human monocyte/ 


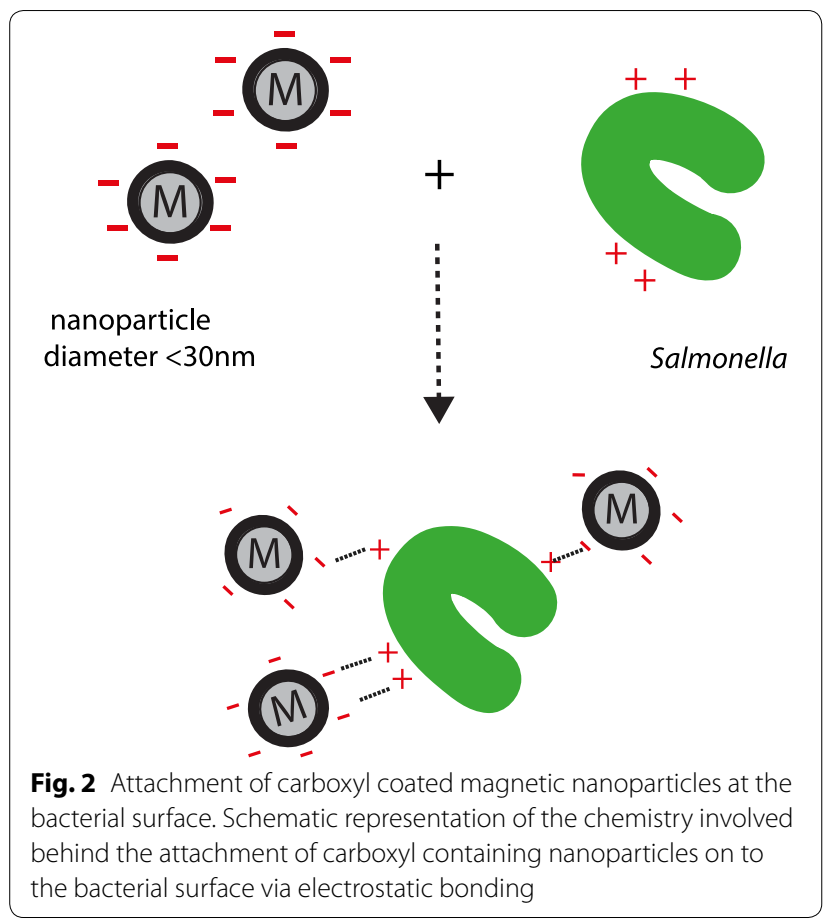

macrophage-like cells (ATCC TIB-202), or processed for transmission electron microscopy.

\section{Paramagnetic labeling of Salmonella does not impair bacterial viability or invasion capabilities}

To verify that the attachment or interaction of Salmonella with the nanoparticles had no significant effect on the viability of the bacteria, we determined the colony forming units (c.f.u) of Salmonella before and after incubation with magnetic nanoparticles at $37{ }^{\circ} \mathrm{C}$. Sonicated nanoparticles were incubated with bacterial suspensions at a ratios of 5:1 or 10:1 (nanoparticles:bacteria) and incubated at $37{ }^{\circ} \mathrm{C}$ for $40 \mathrm{~min}$ with shaking. Samples were taken for c.f.u determinations before and after incubation in order to determine possible effects of the nanoparticles on the viability of Salmonella. As shown in Fig. 3a, incubation with nanoparticles had no significant effects on their c.f.u indicating that the attachment of these paramagnetic nanoparticles was not detrimental to the bacteria.

We then determined whether the attachment of nanoparticles to the Salmonella surface would affect the invasion process of Salmonella. For this purpose, we infected non-phagocytic, intestinal epithelial cells, with either non-tagged Salmonella or nanoparticle-tagged bacteria at an MOI of 5:1 (bacteria:epithelial cells). Invasion ratios were determined by calculating the total intracellular bacteria $2 \mathrm{~h}$ post infection (p.i) for the control group and the test group. As shown in Fig. 3b, no significant differences were observed on the invasion rates of Salmonella, indicating that the attachment of the nanoparticles did not physically hinder the invasion/uptake process of Salmonella in into intestinal epithelial cells. Furthermore, following invasion, intracellular Salmonella retained the attached paramagnetic nanoparticles within the Salmonella containing vacuole. As shown in Fig. 3c (i), the magnetic nanoparticles showed close association with the bacterial outer surface. As expected for electrostatic interactions with only limited positively charged regions of the bacterial surface, the nanoparticles were not uniformly distributed over the entire bacterial surface, but rather found at only a few localized sites.

\section{SCV purification and analysis}

Following internalization into the host cell, Salmonella resides within a membrane-bound compartment referred to as the Salmonella-containing vacuole (SCV), the maturation of which requires bacterial effector proteins [19]. The maturation of the SCV into a replicative niche can be followed by the acquisition and/or exclusion of various host cell proteins [20]. At early stages post-invasion, endosomal markers such as Rab5 and early endosome antigen 1 (EEA1) are found at the SCV membrane [21]. As the SCV continues to mature, it acquires late endosomal and lysosomal markers such as LAMP1, some of which are involved in acquisition of nutrients from the host cell cytosol [22].

In order to determine whether pre-labeling of Salmonella with paramagnetic nanoparticles might affect association of host cell proteins with the SCV, we infected the THP-1 human macrophage-like cell line with labeled bacteria, and at different time points postinfection, cells were fixed and immuno-stained with anti-Rab5 and anti-LAMP-1 antibodies, both standard markers for the SCV membrane [21]. As depicted in Fig. 4a, nanoparticle-labeled Salmonella showed co-localization with both Rab5 and LAMP-1, indicating that the bacteria reside within a phagosome-like vacuole indistinguishable from the $\mathrm{SCV}$, at least with regard to these standard markers [21]. Both Rab5 and LAMP-1 are also well-characterized markers for early endosomes and lysosomes. In order to rule out the possibility that nanoparticle-bound, intracellular Salmonella might be affected in SCV maturation and fail to avoid fusion with lysosomes, we chose to use an additional marker specific for lysosomes but not for the SCV [22]. Lysosomal integral membrane protein-2 (LIMP-2), is a member of CD36 superfamily of scavenger receptors [23] and plays an important role in lysosomal membrane organization. LIMP-2 serves as a receptor for lysosomal mannose-6-phosphateindependent targeting of $\beta$-glucocerebrosidase [24]. 

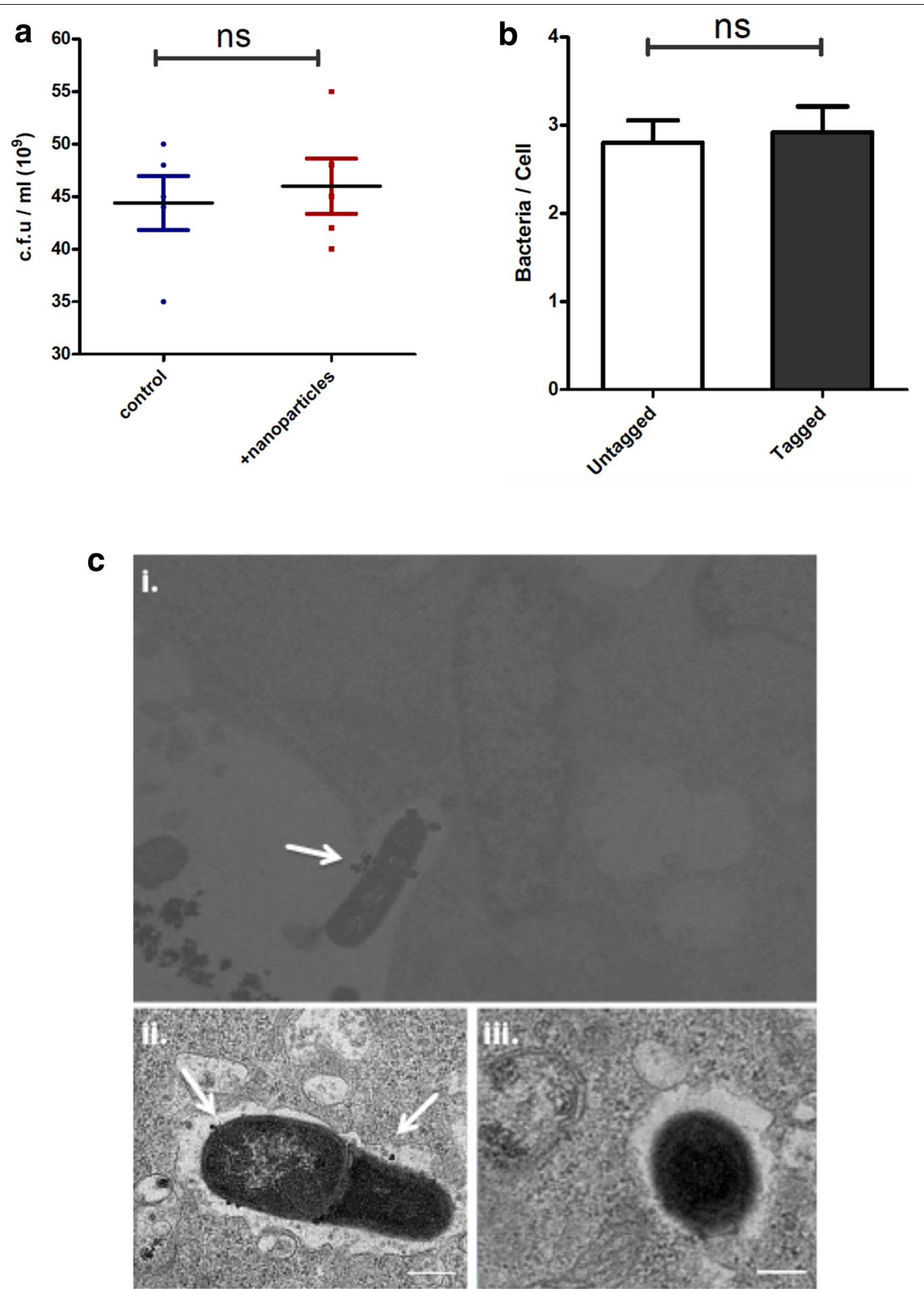

Fig. 3 Nanoparticle attachment does not impair bacterial viability or invasion capability. The attachment of nanoparticles on bacterial surface had no significant effect on the viability of Salmonella as determined by bacterial c.f.u determinations before and after incubation with nanoparticles (a). The nanoparticles did not compromise the ability of Salmonella to invade non-phagocytic, intestinal epithelial cells (b). Representative TEM images showing nanoparticle-bound Salmonella invading THP-1 cells c (i). Post-internalization, the bacteria retain the nanoparticles within the SCV, indicated by arrows $\mathbf{c}$ (ii), in contrast to non-labelled bacteria c (iii) 


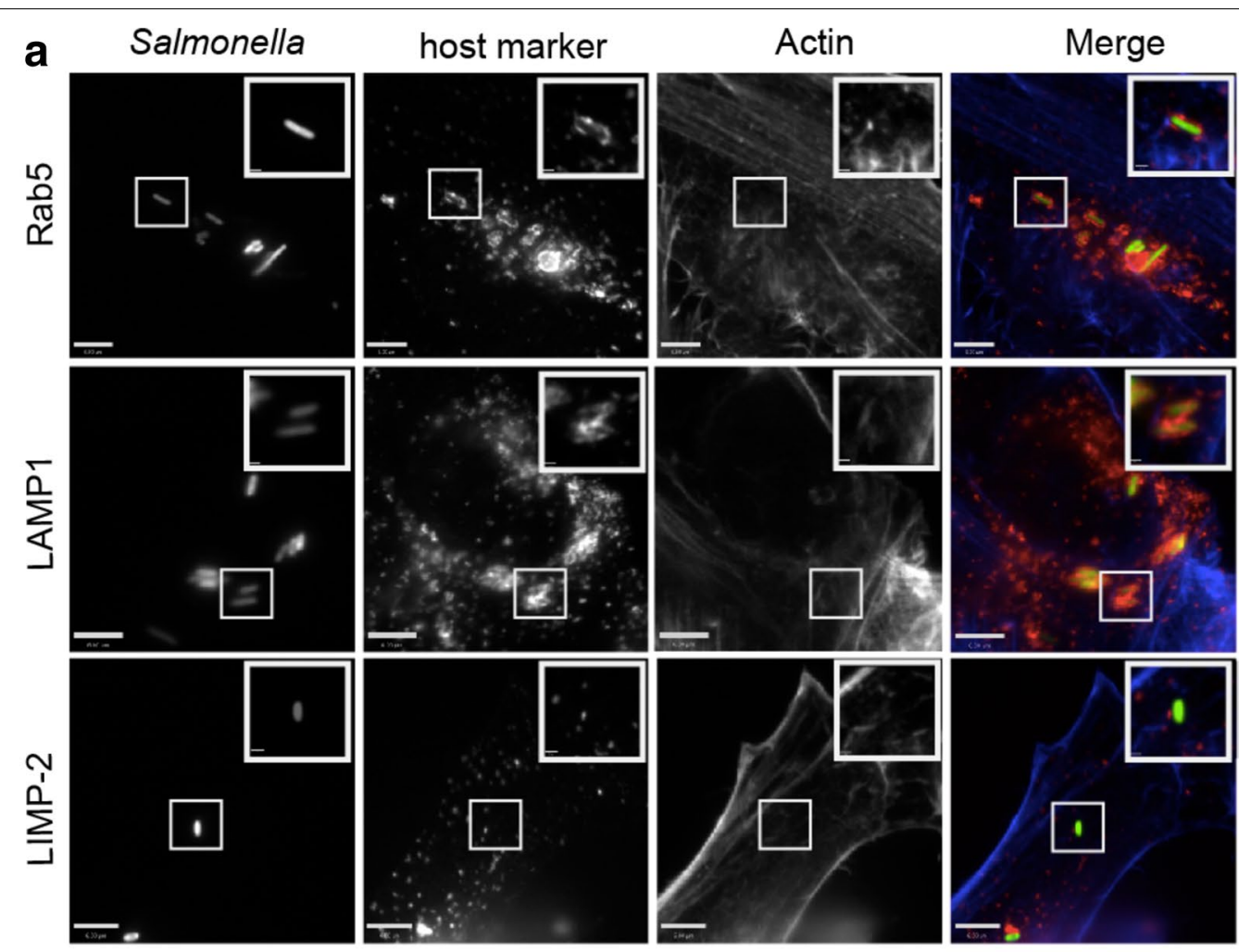

b

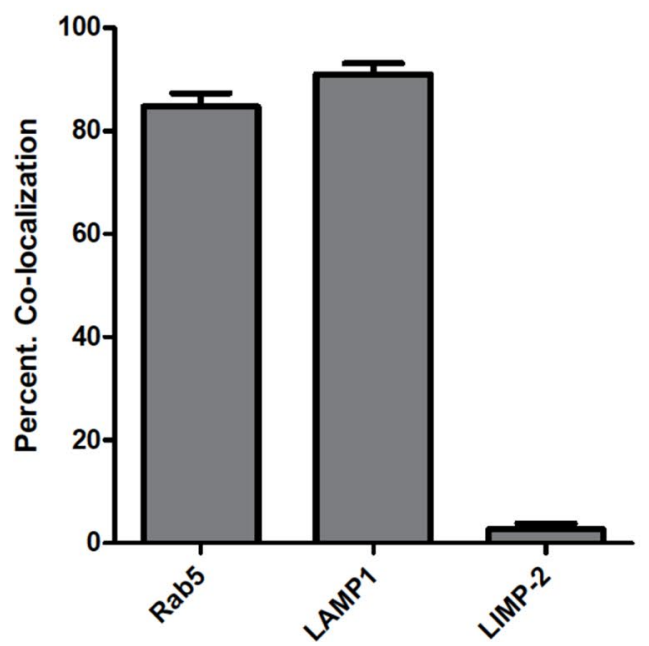

Fig. 4 Co-localization studies. Wild-type Salmonella expressing GFP (green) were used to infect THP-1 macrophage cells grown on coverslips at a multiplicity of infection (MOI) of 5. The monolayers were fixed 10 min post infection, and Rab5 association determined at and after $6 \mathrm{~h}$ and to detect bacterial association with LAMP-1 and LIMP-II. a Green, GFP-expressing bacteria co-localized with both Rab5 (red) and LAMP-1 (red), but not with LIMP-II (red), indicating that Salmonella is present within an SCV and not lysosomes. b Co-localization of intracellular Salmonella with both Rab5 and LAMP-1 were also quantified using the Velocity quantification software. The images shown are a representative of at least 25 individual cells per condition performed in duplicates on at least three independent occasions

As shown in Fig. 4a, intracellular Salmonella does not co-localize with anti-LIMP-2 antibodies thus ruling out lysosomal interaction with the SCV.
Finally, to determine whether pre-labeling of Salmonella with paramagnetic nanoparticles might be used to purify intracellular Salmonella-containing vacuoles, host 
cells were infected, and later lysed for recovery of intracellular Salmonella in the presence of a magnetic field. Cell lysates were then placed in a magnetic field and nonbound material was carefully removed, discarded and replaced by $1 \mathrm{ml}$ of a $1 \times \mathrm{PBS} / 2 \%$ sucrose solution. The recovered material was then further processed by incubating in the presence of anti-Rab5 and anti-LAMP-1 antibodies for microscopy or subjecting aliquots to SDSPAGE followed by Western blotting analysis. As shown in Fig. 5, GFP-expressing Salmonella recovered in this manner from cell lysates showed exterior labeling with both Rab5 and LAMP-1, consistent with the presence of intact SCV membranes as previously shown in Fig. 4a, where 90-95\% of intracellular Salmonella showed co-localization with both these well characterized SCV markers (Fig. 4b). Control experiments using the secondary antibodies alone showed no co-localization with purified SCVs or labeling of the GFP-expressing Salmonella (Additional file 1: Fig. S1).

\section{Western blot analysis}

To verify the results as observed by microscopic analysis of the purified SCVs, we also performed Western blotting for SCV-associated host proteins using the purified SCV preparations. As a control, nanoparticle-bound Salmonella were heat-killed, and incubated with macrophage for uptake by phagocytosis. The SCV preparations were then subjected to SDS-PAGE followed by Western blotting against various lysosomal markers. As expected, SCV markers such as Rab5 and EEA1 (early SCV markers) and LAMP-1 were shown to be present on purified SCV preparations. As previously reported, LAMP-2A was found to be enriched on purified SCVs compared to heat-killed Salmonella [22]. In contrast, association with LIMP-2 and LC3 was significantly reduced or absent compared to control phagosomes containing dead Salmonella (Fig. 6a). Control preparations using heat-killed Salmonella showed the presence of all lysosomal markers, as expected for mature lysosomes. The presence or absence of these markers both on purified SCVs as well
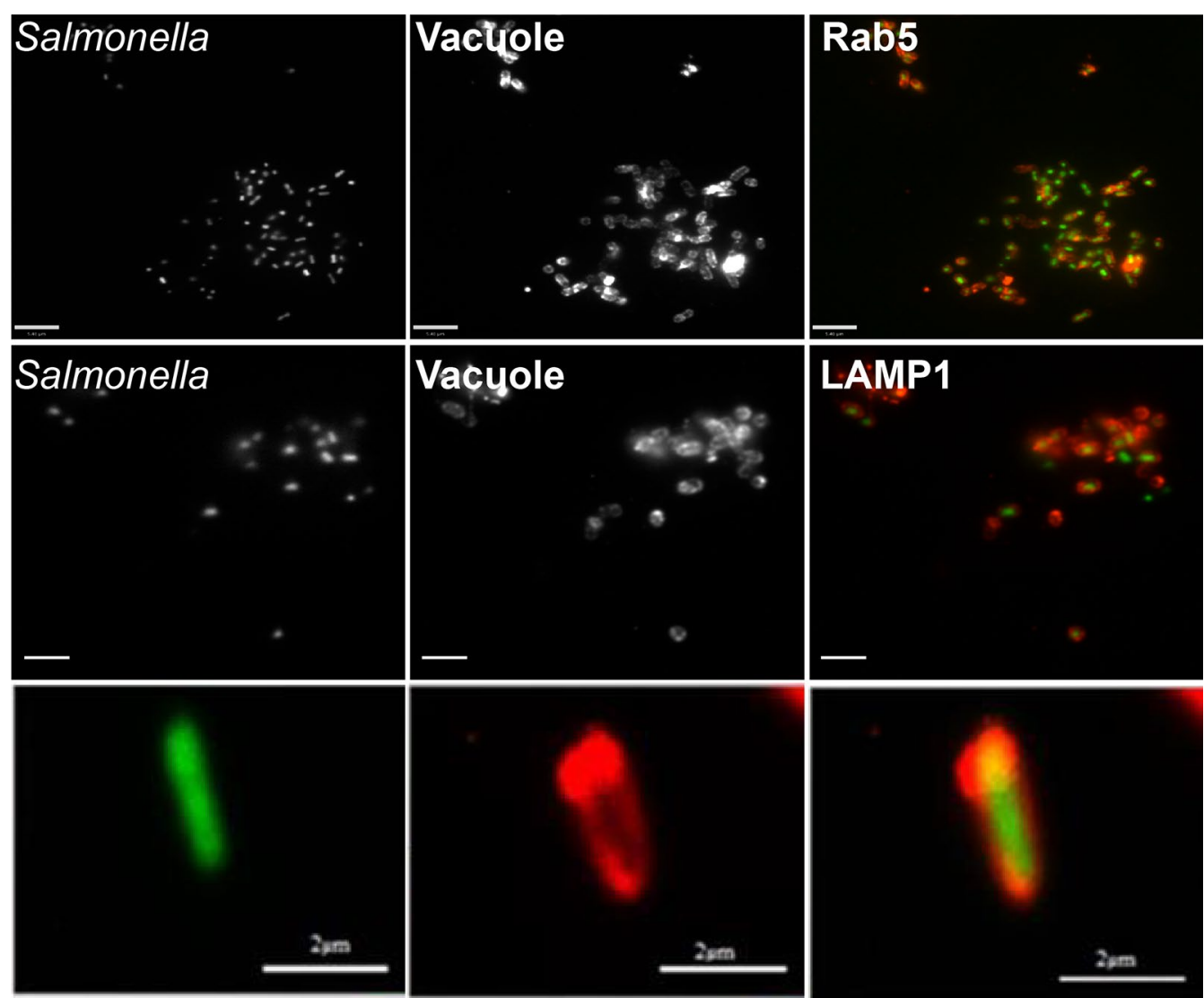

Fig. 5 Microscopic analysis of purified SCVs. Bacteria recovered by application of a magnetic field after lysis of THP-1 macrophages were incubated in presence of antibodies against the standard SCV markers Rab5 and LAMP-1. GFP-expressing Salmonella show exterior labelling with both Rab5 and LAMP-1 indicating intact, isolated SCV. The bottom panel shows a close-up of an individual SCV showing a single bacterium (green), LAMP-1 staining (red) 
as heat-killed Salmonella were also quantified as shown in Fig. 6b. The values in arbitrary units represent band density relative to that of cell lysates.

Finally, we also verified the presence of the Salmonella effector proteins SopE and SopB, both of which have previously been shown to localize to the SCV membrane, and which play an important role in maintaining and modulating the SCV [25-27]. As can be seen in Fig. $6 c$, both secreted effector proteins are also found in the membrane of the SCV. These results indicate that the SCVs purified in this manner represent viable, Salmonella-derived compartments, as the heat-killed Salmonella did not show the presence of these effectors when recovered from macrophage (Fig. 6c).

These results indicated that the purified SCVs showed not only the standard host cell markers associated with the SCV membrane, Rab5 and LAMP-1, but were also essentially devoid of cross-contamination from other endosomal compartments such as lysosomes, as indicated by the absence of LIMP-II or LC3 (Fig. 6a).

\section{Discussion}

The ability of Salmonella to successfully establish an infectious niche and replicate in a wide variety of cell types is linked to the pathogen's capability of residing in a vacuolar compartment and evasion of phagolysosomal clearance $[28,29]$. Our current knowledge of the SCV biogenesis, maturation and interactions with host proteins is largely based on immunofluorescence/ co-localization studies, which is limited by the choice of antibodies [21]. A number of prior studies using gradient centrifugation, immunoprecipitation, or FACS sorting have provided important insights into a number of host proteins with which Salmonella effector proteins interact at the SCV interface [12-14], but a complete characterization has remained elusive due to technical difficulties in the purification. Here we report a rapid, new method to isolate intact SCVs by labeling Salmonella with paramagnetic nanoparticles prior to infection. After gentle disruption of the cell membrane, the SCV can be easily purified using a magnetic field.

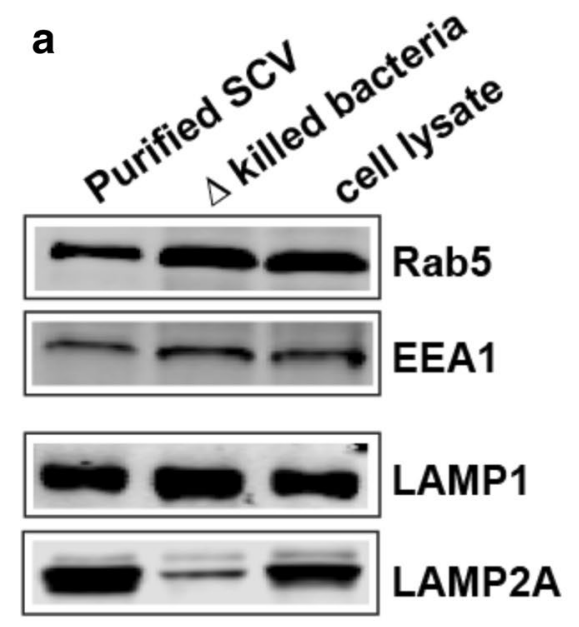

b
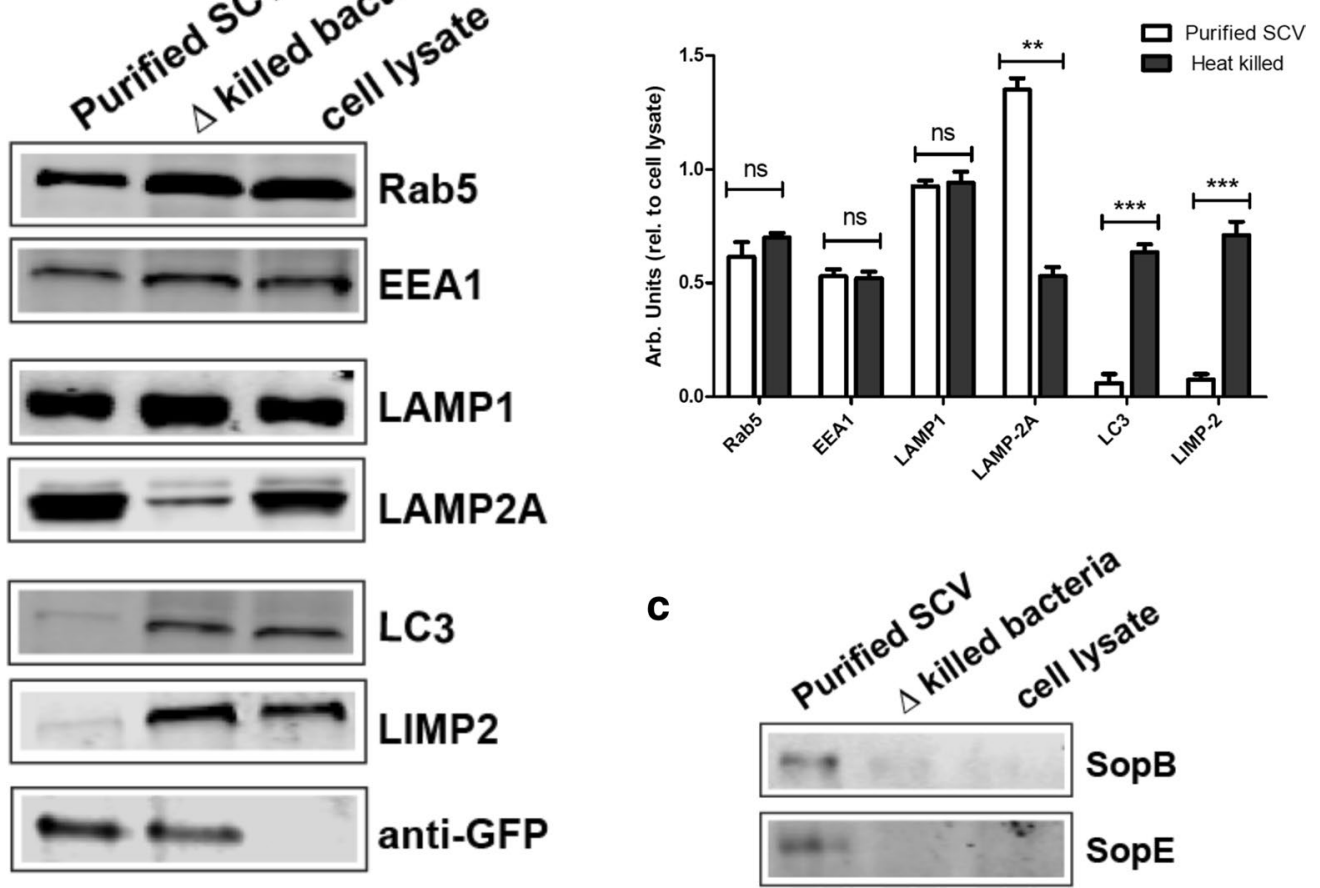

Fig. 6 Western blot analysis of purified SCV. Purified SCVs recovered from THP-1 macrophage infected with either live (SCV) or heat-killed (killed) GFP-expressing Salmonella were subjected to SDS-PAGE and Western blotting using antibodies against the indicated host proteins. Purified SCVs show the presence of both early (Rab5 and EEA1) and late (LAMP-1 and LAMP-2A) SCV markers but not LIMP-II which is present in heat (killed) group a indicating the purified SCVs are devoid of lysosomal contamination. Loading controls consisted of a constant amount of uninfected host cell lysates used as an internal standard for the quantification. $\mathbf{b}$ Quantification of the relative amounts of the indicated host proteins present in SCVs purified from macrophage infected with either live or heat-killed Salmonella. Bacterial effectors SopE and SopB were found exclusively only on purified SCVs (c). Signal intensities were determined relative to a constant amount of non-infected cell lysates. The data shown are the means and standard error of at least two, independent experiments. Statistical significance was determined by a two-way Annova, where $p>0.5$ is considered non-significant (ns); ${ }^{* *} p<0.01 ;{ }^{* * *} p<0.001$ 
The method described here for SCV isolation using paramagnetic nanoparticles is rapid and requires a minimum of manipulations, in contrast to column- or flowassisted cell sorting (FACS)-based methods [14]. This method presents several advantages when compared to the traditional phagosome isolation methods. (i) The process eliminates time-consuming, multiple centrifugation steps, allowing more samples to be handled in less time and yields intact SCVs at high purity. (ii) The method also eliminates the need for specialized and sensitive equipment, such as ultracentrifugation or flow-assisted cell sorting. (iii) The approach also avoids the necessity of covalent labeling of the bacterial outer membrane with ligands using chemical reagents, which may affect the secretion of virulence proteins necessary for establishment of the intracellular niche. As demonstrated in Fig. 3a, the labeling of the nanoparticles does not interfere with Salmonella's ability to invade either intestinal epithelial or macrophage cells. The SCVs purified using paramagnetic nanoparticles showed association with standard SCV markers, Rab5 and LAMP-1 [20, 21, 25], but essentially no association with another lysosomal marker, LIMP-2, confirming minimal or no contamination with lysosomes or other cellular compartments. Furthermore, we could demonstrate the presence of Salmonella effector proteins known to co-localize with SCVs. We suggest the method described here could also be applied for isolation of phagosomes harboring other intracellular bacteria such as Mycobacteria, Legionella, Brucella, etc. This should aid in identification and characterization of host factors associated with the membranes of such intracellular pathogens and which could potentially serve as pharmaceutical targets against intracellular pathogens residing within vacuoles.

\section{Conclusion}

In summary this, method for the isolation of Salmonellacontaining vacuoles (SCV) is specific, rapid, efficient, requires a minimum of specialized equipment, and can be easily adapted to address key biological questions concerning host-pathogen interactions.

\section{Methods}

\section{Cell culture and growth conditions}

Human macrophage like cells THP-1 (DSMZ Cat. Nr. ACC-16 (Tsuchiya et al. [30]) were grown in Iscove's Dulbecco modified Eagle medium (Biochrom), supplemented with $10 \%$ fetal Calf serum (FCS) under standard tissue culture conditions of $37^{\circ} \mathrm{C}, 5 \% \mathrm{CO}_{2}$. The cells were regularly passaged with $1 \%$ trypsin EDTA (Biochrom).

The wild-type, virulent S. typhimurium strain SL1344 (Hoiseth and Stocker [31]) harboring the GFP fusion under control of the rps $M$ promoter [4] was streaked onto LB agar plates containing kanamycin $(50 \mu \mathrm{g} / \mathrm{ml})$ the day before the assays, and a single colony was used to inoculate in LB broth culture was grown to the late $\log$ phase $\left(\mathrm{OD}_{600} \sim 2\right)$. The cells were centrifuged and the resulting pellets were re-suspended in cell culture medium and diluted to obtain the desired MOI.

\section{SCV isolation \\ Labeling Salmonella with paramagnetic nanoparticles}

From a stock solution of $3 \mathrm{mg} / \mathrm{ml}$ of carboxyl-coated, paramagnetic cobalt nanoparticles of diameter $10-50 \mathrm{~nm}$ (TurboBeads, Zurich), a $0.5 \mathrm{ml}$ aliquot was sonicated to disperse aggregates, followed by centrifugation at $1000 \times g$ for $30 \mathrm{~s}$. Following centrifugation, $0.1 \mathrm{ml}$ of the resulting supernatant was diluted 1:10 in sterile, de-ionized water prior to use. Salmonella strains grown in LB as described above were collected from $1 \mathrm{ml}$ of culture by centrifugation in a microfuge at $16,000 \times g$ for $5 \mathrm{~min}$. The resulting bacterial pellets were re-suspended in PBS and incubated in the presence of a 5:1 ratio of nanoparticles:bacteria at $37^{\circ} \mathrm{C}$ for $20 \mathrm{~min}$, with shaking. The bacterial suspension was then filtered through a $0.2 \mu \mathrm{m}$ filter fitted to a $5 \mathrm{ml}$ syringe. The filters were washed twice with two volumes of PBS, then the filter was inverted and the bacteria recovered in $1 \mathrm{ml}$ of PBS collected into a sterile microfuge tube, and used either for infection or processed for electron microscopy.

\section{Infection and recovery of bacteria}

THP-1 human macrophage cells grown in a 6-well cell culture plate were infected at a cell density of $10^{6}$ cells/ well with the nanoparticle-labeled Salmonella $(\mathrm{MOI}=5)$ and incubated at $37{ }^{\circ} \mathrm{C}, 5 \% \mathrm{CO}_{2} .24 \mathrm{~h}$ post-infection, the cell culture media was removed; infected cells were washed twice with PBS then lysed by addition of $1 \mathrm{ml} /$ well of cell lysis buffer ( $0.1 \%$ Triton X-100 in water). The resulting cell lysates were then placed in a magnetic field (Single Place Magnetic Stand, Ambion, USA) and the supernatant was carefully removed and discarded, and replaced by $1 \mathrm{ml}$ of a $1 \times \mathrm{PBS} / 2 \%$ sucrose solution. The buffer was again removed and replaced by $0.1 \mathrm{ml}$ of the same buffer to concentrate the SCVs, which were then used either for staining with antibodies or subjected to SDS PAGE and Western blotting.

\section{Western blots}

Isolated SCV's were centrifuged and pelleted, followed by boiling the samples in Laemmli $(6 \times)$ buffer. Equal volumes were loaded onto $12 \%$ SDS PAGE and transferred to nitrocellulose membrane (GE Healthcare). The membrane was blocked in 3\% skim milk powder in 
$1 \times$ TBS, followed by incubation in appropriate primary antibodies and HRP conjugated secondary antibodies at the above mentioned dilutions. Signals were revealed using the ECL detection system kit (Thermo scientific pierce).

\section{Confocal microscopy}

THP-1 cells were seeded onto $12 \mathrm{~mm}$ sterile coverslips (Carl Roth), infected with Salmonella strains expressing GFP at an MOI of 5. $24 \mathrm{~h}$ post-infection, the cells were washed in pre-warmed $1 \times \mathrm{PBS}$ and fixed with PBS $/ 4 \%$ paraformaldehyde (PFA, Sigma) for $15 \mathrm{~min}$, permeabilized with $0.1 \%$ Triton X-100 (Sigma-Aldrich) in PBS for 5 min, blocked with PBS/1.5\% BSA (sigma) for $1 \mathrm{~h}$ and incubated with the appropriate antibodies for $1 \mathrm{~h}$ or overnight at $4{ }^{\circ} \mathrm{C}$. The cells were washed with $1 \times$ PBS and incubated with appropriate secondary antibodies for $1 \mathrm{~h}$ at room temperature. The coverslips were mounted with Mowiol (Sigma-Aldrich) onto glass slides (Carl Roth) and were visualized using a Leica SP-II laser scanning confocal microscope (LCSM) using a $63 \times$ oil immersion objective.

Isolated SCVs were incubated for $1 \mathrm{~h}$ with specific primary antibodies in $1.5 \mathrm{ml}$ microfuge tubes (Eppendorf) and placed in a magnetic stand. The supernatant was removed by pipetting along the opposite wall of the of the microfuge tube (relative to the magnetic stand), and the SCVs were gently washed with $1 \times \mathrm{PBS} / 2 \%$ sucrose solution, followed by incubation with suitable secondary antibody in the same microfuge tubes for 1 h. After re-placement in the magnetic stand, the samples were again washed and mounted onto glass slides for microscopic visualization.

\section{Transmission electron microscopy}

THP-1 cells were infected with either tagged or nontagged Salmonella, and the cells were then immediately fixed by addition of $1.5 \%$ PFA and $1.5 \%$ glutaraldehyde in $0.15 \mathrm{M}$ sodium cacodylate buffer, $\mathrm{pH}$ 7.4. After incubation at room temperature for $1 \mathrm{~h}$, the cell suspensions were post-fixed for $2 \mathrm{~h}$ at $4{ }^{\circ} \mathrm{C}$ in $1 \%$ osmium tetroxide in sodium cacodylate buffer, and subsequently dehydrated in a series of ethanol steps, and then further processed with acetone prior to Epon embedding. Sections were cut with a microtome and mounted on Formvar-coated copper grids. The sections were postfixed with uranyl acetate and lead citrate and examined under the electron microscope.

\section{Additional file}

Additional file 1: Figure S1. In vitro grown, nanoparticle-labeled Salmonella does not show exterior labeling with host cell lysosomal marker antibodies. Paramagnetic nanoparticle-tagged, GFP-labeled Salmonella were incubated with anti-Rab5, LAMP-1 and LIMP-II antibodies for $1 \mathrm{~h}$ at room temperature, followed by a wash with $1 \times \mathrm{PBS}$, and subsequently stained with the Alexa-Flour 594 labelled secondary antibodies. In vitro, culture-grown GFP-expressing Salmonella does not show labelling with any of the host cell markers.

\section{Abbreviations \\ SCV: Salmonella-containing vacuole; MOI: multiplicity of Infection; CFU: colony forming unit; LAMP1: lysosomal associated membrane protein 1; LC3: microtubule-associated protein 1A/1B-light chain 3.}

\section{Authors' contributions}

VS and KT carried out the experimental work, PS maintained the bacterial and cell culture stocks. VS and KT analyzed the data and wrote the manuscript. KT supervised the entire project. VS and KT were involved in the design of all experiments and revised the manuscript. All authors read and approved the final manuscript.

\section{Acknowledgements}

Authors gratefully acknowledge the Humboldt University, Parasitology Department Microscopy Facility, Dr. Maik Lehmann for helping with the electron microscopy. The authors would also like to acknowledge Dr. Arthur Thompson for helpful discussions.

\section{Competing interests}

The authors declare that they have no competing interests.

\section{Availability of data and materials}

All data generated or analysed during this study are included in this published article (and its additional files).

\section{Consent for publication}

Not applicable.

\section{Ethics approval and consent to participate}

Not applicable.

\section{Funding}

This work was supported by Deutsche Forschungsgemeinschaft (DFG) through Priority Program SPP1136 Grant TE416/1-1 (KT), Graduate Research Training Grant GRK1121 (to KT and VS), and the German Ministry for Education and Research (Bundesministerium für Bildung und Forschung, BMBF) InfectControl 2020 (IRMRESS) grant 03ZZ0805A (KT).

\section{Publisher's Note}

Springer Nature remains neutral with regard to jurisdictional claims in published maps and institutional affiliations.

Received: 11 May 2018 Accepted: 3 July 2018

Published online: 31 July 2018

\section{References}

1. Lahiri A, lyer N, Das P, Chakravortty D. Visiting the cell biology of Salmonella infection. Microbes Infect. 2010;12:809-18.

2. Singh Vikash. Salmonella serovars and their host specificity. J Vet Sci Anim Husb. 2013;1:301. 
3. Chessa D, Winter MG, Jakomin M, Baumler AJ. Salmonella enterica serotype typhimurium std fimbriae bind terminalalpha $(1,2)$ fucose residues in the cecal mucosa. Mol Microbiol. 2009;71:864-75.

4. Vazquez-Torres SA, Jones-Carson J, Bäumler AJ, Falkow S, Valdivia R, Brown W, Le M, Berggren R, Parks WT, Fang FC. Extraintestinal dissemination of Salmonella by CD18-expressing phagocytes. Nature. 1999;401:804-8.

5. Galan JE. Interactions of Salmonella with host cells: encounters of the closest kind. Proc Natl Acad Sci. 1998;95:14006-8.

6. Raffatellu M, Wilson RP, Chessa D, Andrews-Polymenis H, Tran QT, Lawhon S, Khare S, Adams LG, Bäumler AJ. SipA, SopA, SopB, SopD, and SopE2 contribute to Salmonella enterica serotype Typhimurium invasion of epithelial cells. Infect Immun. 2005;73:146-54.

7. Hensel M, Shea JE, Waterman SR, Mundy R, Nikolaus T, Banks G, et al. Genes encoding putative effector proteins of the type III secretion system of Salmonella pathogenicity island 2 are required for bacterial virulence andproliferation in macrophages. Mol Microbiol. 1998;30:163-74.

8. Smith AC, Heo WD, Braun V, Jiang X, Macrae C, Casanova JE, et al. A network of Rab GTPases controls phagosome maturation and is modulatedby Salmonella enterica serovar Typhimurium. J Cell Biol. 2007:176:263-8.

9. Bakowski MA, Braun V, Brumell JH. Salmonella-containing vacuoles: directing traffic and nesting to grow. Traffic. 2008;9:2022-31.

10. Desjardins M, Huber LA, Pardon RG, Griffiths G. Biogenesis of phagolysosomes proceeds through a sequential series of interactions with the endocytic apparatus. J Cell Biol. 1994;124:677-88.

11. Desjardins $M$, Celis JE, van Meer $G$, Dieplinger $H$, Jahraus A, Griffiths G, Huber LA. Molecular characterization of phagosomes. J Biol Chem. 1994;269:32194-200.

12. Diettrich O, Mills K, Johnson AW, Hasilik A, Winchester BG. Application of magnetic chromatography to the isolation of lysosomes from fibroblasts of patients with lysosomal storage disorders. FEBS Lett. 1998:441:369-72.

13. Perrin-Cocon LA, Marche PN, Villiers CL. Purification of intracellular compartments involved in antigen processing: a new method based on magnetic sorting. Biochem J. 1999;338:123-30.

14. Luhrmann A, Haas A. A method to purify bacteria-containing phagosomes from infected macrophages. Methods Cell Sci. 2000;22:329-41.

15. Vaudaux P, Waldvogel FA. Gentamicin antibacterial activity in the presence of human polymorphonuclear leukocytes. Antimicrob Agents Chemother. 1979;16:743-9.

16. Dickson JS, Koohmaraie M. Cell surface charge characteristics and their relationship to bacterial attachment to meat surfaces. Appl Environ Microbiol. 1989:55:832-6.

17. Hermansson M, Kjelleberg S, Korhonen TK, Stenström TA. Hydrophobic and electrostatic characterization of surface structures of bacteria and its relationship to adhesion to an air-water interface. Arch Microbiol. 1982;131:308-12.
18. Grass RN, Athanassiou EK, Stark WJ. Covalently functionalized cobalt nanoparticles as a platform for magnetic separations in organic synthesis. Angew Chem Int Ed. 2007;46:4909-12.

19. Cain RJ, Hayward RD, Koronakis V. Deciphering interplay between Salmonella invasion effectors. PLoS Pathog. 2008;4:1000037.

20. Drecktrah D, Knodler LA, Howe D, Steele-Mortimer O. Salmonella trafficking is defined by continuous dynamic interactions with the endolysosomal system. Traffic. 2007:8:212-25.

21. Steele-Mortimer O. The Salmonella-containing vacuole-moving with the times. Curr Opin Microbiol. 2008;11:38-45.

22. Singh V, Finke-Isami J, Hopper-Chidlaw AC, Schwerk P, Thompson A, Tedin K. Salmonella co-opts host cell chaperone-mediated autophagy for intracellular growth. J Biol Chem. 2017;292:1847-64.

23. Brumell JH, Grinstein S. Salmonella redirects phagosomal maturation. Curr Opin Microbiol. 2004;7:78-84.

24. Febbraio M, Hajjar DP, Silverstein RL. CD36: a class B scavenger receptor involved in angiogenesis, atherosclerosis, inflammation, and lipid metabolism. J Clin Invest. 2001;108:785-91.

25. Reczek D, Schwake M, Schroder J, et al. LIMP-2 is a receptor for lysosomal mannose-6-phosphate independent targeting of beta-glucocerebrosidase. Cell. 2007;131:770-83.

26. Zhou D, Chen LM, Hernandez L, Shears SB, Galan JE. A Salmonella inositol polyphosphatase acts in conjunction with other bacterial effectors to promote host cell actin cytoskeleton rearrangements and bacterial internalization. Mol Microbiol. 2001;39:248-59.

27. Mason D, Mallo GV, Terebiznik MR, Payrastre B, Finlay BB, Brumell JH, Rameh L, Grinstein S. Alteration of epithelial structure and function associated with Ptdlns(4,5)P2 degradation by a bacterial phosphatase. J Gen Physiol. 2007;129:267-83.

28. Eswarappa SM, Negi VD, Chakraborty S, Chandrasekhar Sagar BK, Chakravortty D. Division of the Salmonella-containing vacuole and depletion of acidic lysosomes in Salmonella-infected host cells are novel strategies of Salmonella enterica to avoid lysosomes. Infect Immun. 2010;78(1):68-79.

29. Terebiznik MR, Vieira OV, Marcus SL, Slade A, Yip CM, Trimble WS, Meyer T, Finlay BB, Grinstein S. Elimination of host cell Ptdlns $(4,5) P(2)$ by bacterial SigD promotes membrane fission during invasion by Salmonella. Nat Cell Biol. 2002;4:766-73

30. Tsuchiya S, Kobayashi Y, Goto Y, Okumura H, Nakae S, Konno T, Tada K. Induction of maturation in cultured human monocytic leukemia cells by a phorbol diester. Cancer Res. 1982;42:1530-6.

31. Hoiseth SK, Stocker BAD. Aromatic-dependent Salmonella typhimurium are non-virulent and effective as live vaccines. Nature. 1981:291:238-9.
Ready to submit your research? Choose BMC and benefit from:

- fast, convenient online submission

- thorough peer review by experienced researchers in your field

- rapid publication on acceptance

- support for research data, including large and complex data types

- gold Open Access which fosters wider collaboration and increased citations

- maximum visibility for your research: over $100 \mathrm{M}$ website views per year

At BMC, research is always in progress.

Learn more biomedcentral.com/submissions 